\title{
On the Most Probable Value of the Solar Parallax.
}

The interesting discussion of the present state of our knowledge of the Solar parallax given by Dr. Boris Weinberg, of the University of Odessa, in A. N. 3866, induces me to add a few lines in explanation of the value

$$
\pi=8.796 \pm 0.006
$$

adopted in 1895 and used uniformly in my papers on the dimensions of the Planets and Satellites of the Solar System published in the A. N. during the past three years. This value was deduced by the writer while at Chicago, from a discussion of the various determinations of the Solar parallax then available. As the paper dealing with this investigation was never published, and the original manuscript perished in the fire at Flagstaff, Arizona, Sept. 141897 , it is difficult at this time to give the details of the computations. Yet it is easy to recall assigning the greatest weight to the aberration method, and to that depending on the opposition of Mars and the small planets. In general the weights were assigned from the inherent consistency of the several determinations by a given method, and also from the general validity of the several methods as they appeared to the investigator.

Some two years later Professor Newcomb obtained in his concluding work on the Constants of Astronomy the value

$$
\pi=8: 7965 \pm 0.0045
$$

(cf. Astronomical Constants, p. 166). This value is freed from systematic errors, so far as Professor Newcomb was able to effect their elimination. The agreement between the values (a) and (b), and Weinberg's value

$$
\pi=8.8004 \pm 0.00243
$$

founded upon a new method of adjustment by the theory of pro. bability is sufficiently striking to render comment unnecessary. Dr. Weinberg does not seem to have included the values which result from Professor C. L. Doolittle's recent determinations of the constant of Aberration at the Flower Observatory, combined with the velocity of light. Doolittle's careful work extends over several years and will yield one of the most refined determinations of the Aberration constant ever made. The mean value up to the time is about

$$
20.530
$$

which combined with the Michelson-Newcomb velocity of light (namely, $V=299860 \pm 30 \mathrm{~km}$ ) gives for the solar parallax $\pi=8.777$. It may also be noticed that the Aberration constant found by Professor A. Hall, jr., from a careful discussion of the Ann Arbor Meridian Observations of Polaris in 1898 was 20.55 , corresponding to $\pi=8.769$. Accordingly it appears that the values of the solar parallax resulting from recent work on the constant of Aberration are slightly smaller than Weinberg's mean value, and the effect of including them would be to reduce his solar parallax a little. It is regretable that he does not use the method depending on the Constant of Aberration in his discussion; and it might be worth while to apply to the Aberration Method the same theory of adjustment which has been used in case of the other methods for finding the solar parallax.

$$
\text { T. F. F. See. }
$$

\section{Mass of Mercury deduced from Hill's extension to the four Inner Planets of Laplace's Law for the density of the Earth, with a correction to the Mass of Mercury found by the same method in A. N. 3743.}

By $T . \mathcal{F} . \mathcal{F}$. See.

The values of the mass of Mercury heretofore in use are affected with such great uncertainty that an astronomer occupied with problems of Celestial Mechanics is justified in putting forth any reasonable effort which may lead to improvement in our knowledge of this interesting element. Since the publication of the paper in A. N. 3743 , the subject has been reinvestigated, additional measurements of diameter being employed and the other data improved as much as possible.

The result is the deduction by Hill's method of a mass which appears to me as good as this method is capable of producing in the present state of Astronomy; and so far as this method is concerned may be regarded as definitive.

This reexamination of the subject, however, has led to the discovery that the value of the mass given in $A . N$. 3743 , is vitiated by errors of computation or of copying, of such proportions as to make necessary a repetition of the former calculation. The defect thus brought to light assumed in my eyes a very serious aspect, though the resulting change in the reciprocal of the mass of Mercury is less than the uncertainty attaching to the value there deduced, which I had estimated at 1000000 . Under the circumstances it seems advisable to give the corrected data of the former investigation along with the similar data for the mass of Mercury now definitively adopted. The differences thus developed may be useful in showing approximately the degree of uncertainty still attaching to the mass of Mercury, as found by Hill's extension to the four Inner planets of Laplace's law for the density of the Earth (cf. A. N. 3743; A. J. 452, 453). In the present state of Astronomy this method for finding the mass of Mercury has much to commend it, and its applicability appears to be emphasized by the densities since found for the four Galilean Satellites of Jupiter and for Titan, the principal Satellite of Saturn (cf. A. N. 3764).

More recent measurements of the diameter of Mercury seemed to increase somewhat the value $(5.90)$ given in 